\title{
IMPROVED MODEL OF WATER CRESTING
}

\author{
J. F, McCARTHY ${ }^{1}$
}

(Received 5 August 1991; revised 24 March 1992)

\begin{abstract}
An improved model of water cresting towards horizontal wells is presented, using a hodograph solution to the lateral edge drive model with a constant potential boundary at a finite outer radius. In this model, the water crest tends to a horizontal asymptote far from the well, correcting previous approaches which led to the unphysical result of a crest which never levels off but, rather, tends to a parabolic curve. The hodograph solution yields the shape of the free water-oil interface. It also yields integral representations for the lengths of boundary segments, and these have enabled the derivation of an explicit expression for the critical rate in terms of the outer radius. The critical rates calculated using the improved model do not differ significantly from those calculated using previous approaches. The main advantage of the model, therefore, is not a correction to the quantitative predictions of the critical rate, but the removal of physical inconsistencies in the underlying theory.
\end{abstract}

\section{Introduction}

An introduction to the problem of gas and water cresting for oil production from a horizontal well in a thin oil column reservoir was given in a previous paper [8]. Briefly, water cresting is caused by potential gradients being set up in the flowing oil layer, which draw water from the underlying aquifer towards the well (see Figure 1). There is a critical rate of oil production above which the water will break through into the well, leading to a reduced cross-section for oil to flow. Below the critical rate, a stable crest of stationary water exists whose height is determined by the equilibrium between the potential gradients due to the oil flow and the gravity forces due to the density difference between the oil

\footnotetext{
${ }^{1}$ BHP Research - Melbourne Laboratories, P.O. Box 264, Clayton, Vic. 3168, Australia. (C) Australian Mathematical Society, 1993, Serial-fee code 0334-2700/93
} 


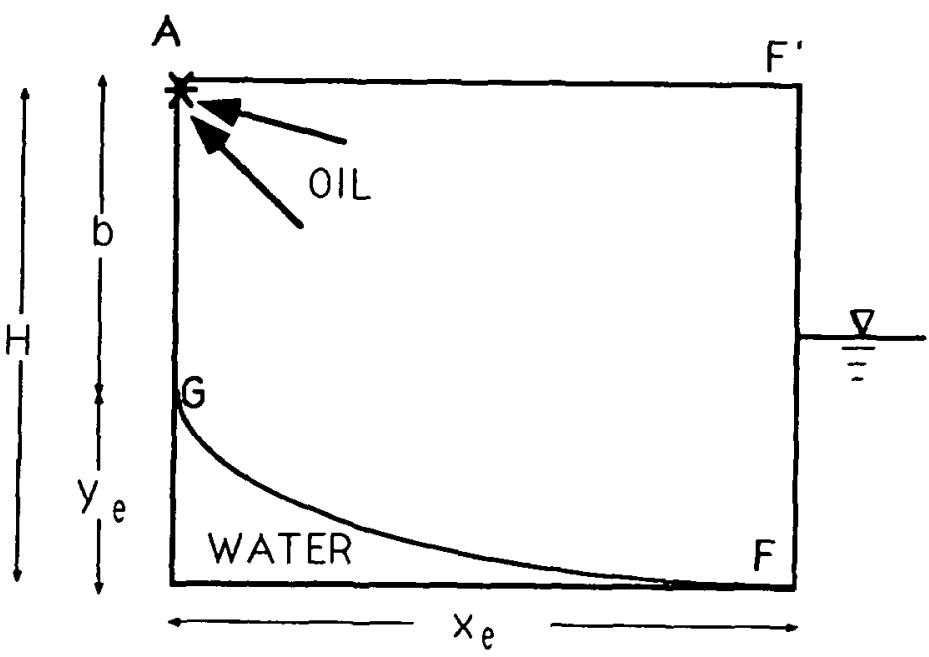

FIGURE 1. Cross-sectional schematic of water cresting towards a line sink on an impermeable plane in an oil reservoir with a constant potential boundary at the outer radius.

and water. The critical rate is the flowrate corresponding to the highest stable crest.

Analytical studies of water cresting $[3,4,6]$ are almost all based on the reservoir geometry and boundary conditions illustrated in Figure 1. The well is represented by a sink $A$ of strength $Q$ situated on a horizontal impermeable plane $\overline{A F^{\prime}}$ above an oil layer. By modelling only a cross-section of the reservoir, we are essentially considering a section of an infinite horizontal well. The oil flows between no-flow boundaries, delimited by the impermeable plane above and the water-oil interface $\overline{G F}$ below. It is assumed that the outer boundary is a constant pressure potential boundary at which the fluid interfaces are fixed and horizontal. This implies that fluids (including oil) are automatically fed into the reservoir at the outer boundary to compensate for production. Under this scenario, called "the lateral edge drive model", it is possible to find stationary solutions to the governing equations. The problem is complicated by the interdependence of the oil-zone potential distribution and the shape of the water crest. It is a free-boundary problem.

Two main approaches have been used to calculate crest shapes and critical rates for the lateral edge drive model:

(i) Giger [6] uses the hodograph method to solve the free boundary problem exactly in the case in which $F^{\prime}=F=\infty$ (see Figure 1); 
(ii) Chaperon [3] uses a steady-state flow potential derived from a solution given by Houpert [7] for the case of an infinite row of vertical wells, and introduces the water crest in a hybrid manner at a later stage. This approach is equivalent to that used by Muskat and Wyckoff [9] in their classic paper on coning towards vertical wells.

The work of Giger and Chaperon will be reviewed in Section 2. Their results are qualitatively very similar. Both predict that the non-dimensionalised critical rate $Q^{*}$ varies approximately inversely with the ratio of the finite outer radius to the height of the reservoir, $x_{e} / h$. This is because of linear flow in the larger part of the reservoir. Both predict a crest that will never level off at a constant height far away from the well. In fact, the crest approaches a parabolic shape far from the well, and therefore has no asymptote. In order to introduce a height for the reservoir and to calculate critical rates the crest is simply truncated at the outer radius, intersecting the initial water-oil contact at a non-zero angle. This non-zero contact angle is unphysical and poses a serious problem for the lateral edge drive model. It has led to skepticism about the ability of the model to predict useful quantitative results. Giger recommends that the use of the model be avoided for any purpose other than a study of the local deformation of the water-oil contact below the horizontal well.

These problems arise because of the use of unphysical boundary conditions for the potential distribution. It is assumed that the flow tends to a uniformly linear distribution at an infinite distance from the well, whereas it is actually required that there be a constant potential boundary at a finite outer radius. In this paper, we show that by proper implementation of the boundary conditions, the hodograph method can be used to give a model in which the water crest tends to a horizontal asymptote at a finite outer radius. An expression for the critical rate is derived and compared with the results derived by Giger and Chaperon.

The layout of this paper is as follows: Section 2 contains a review of the lateral edge drive model as implemented by Giger and Chaperon; Section 3 contains the hodograph solution of the improved model and the results for critical rates and crest shapes; Section 4 contains the conclusion.

\section{Review of the lateral edge drive model}

Darcy's equation for flow in porous media is

$$
V=-\frac{\kappa}{\mu} \nabla(\gamma y+p) \text {, }
$$


where $V$ is the velocity, $\mu$ the viscosity, and $\gamma$ the specific gravity of the flowing fluid; $\kappa$ is the permeability of the porous medium; $y$ is the elevation above some arbitrary level; and $p$ is the pressure. This is often written (see, e.g., [1])

$$
q=-\nabla \phi
$$

where $q=V / \gamma$ is the specific discharge vector and $\phi$, the potential function, is given by

$$
\phi=\kappa(y+p / \gamma) / \mu .
$$

The horizontal and vertical components, $u$ and $v$, respectively, of the specific discharge vector are given by

$$
\begin{gathered}
u=-\partial \phi / \partial x, \\
v=-\partial \phi / \partial y .
\end{gathered}
$$

The hodograph plane, which we denote by $\omega$, is defined by

$$
\omega=u-i v .
$$

In the hodograph plane, the interface between two immiscible liquids of different densities (e.g. oil and water) is given by [1]

$$
u^{2}+(v-K / 2)^{2}=(K / 2)^{2}
$$

where

$$
K=\frac{\kappa}{\mu_{o}} \frac{\left(\gamma_{w}-\gamma_{o}\right)}{\gamma_{o}},
$$

$\gamma_{o}$ and $\gamma_{w}$ are the specific gravities of the oil and water, respectively, and $\mu_{o}$ is the viscosity of the oil.

Consider the physical situation illustrated in Figure 1. This represents the lateral edge drive model, as discussed in the introduction, with the required physical boundary condition of a constant potential boundary at the outer radius, $\overline{F^{\prime} F}$. Giger [6] used the hodograph method to solve the free-boundary problem exactly in the case in which $F^{\prime}=F=\infty$.

The following steps summarise the hodograph method for flow in porous media $[1,2]$ :

(i) the flow region in the coordinate $z$-plane is mapped onto the complex potential $f$-plane $(f=\phi+i \psi)$ by mapping its boundaries;

(ii) the $z$-plane is mapped onto the hodograph $\omega$-plane; 
(iii) the $\omega$-plane is mapped onto the $\bar{\omega}$-plane by using an inversion transformation

$$
\bar{\omega}=K / \omega
$$

(iv) the flow region in both the $\bar{\omega}$ and $f$-planes is mapped onto the upper half of an auxiliary $\zeta$-plane;

(v) with $\zeta$ as an independent parameter, the relation

$$
\frac{d z}{d \zeta}=-\frac{\bar{\omega}(\zeta)}{K} \frac{d f}{d \zeta}
$$

is integrated to find the lengths and shapes of boundary segments in the $z$-plane.

(a)

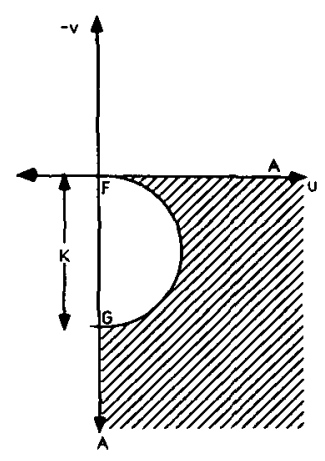

(b)

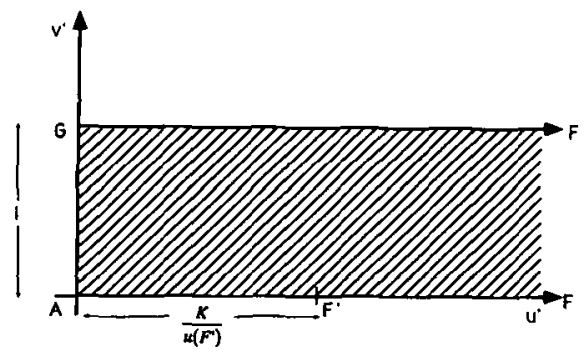

(c)

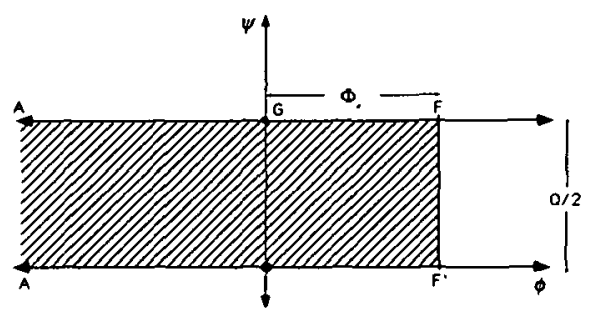

(d)

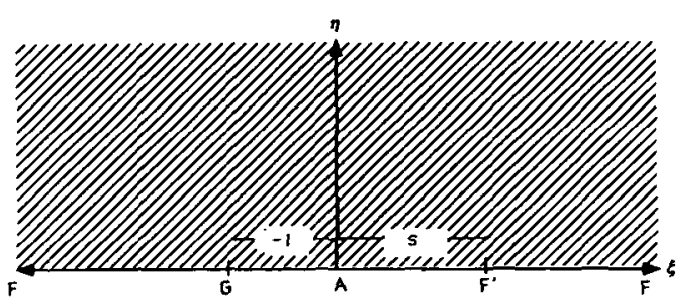

FIGURE 2. Mapping of the flow region onto (a) the hodograph $\omega$-plane, (b) the $\bar{\omega}$-plane and (c) the complex potential $f$-plane. (d) shows the intermediate $\zeta$-plane. 
Figure 2(a) shows the mapping onto the hodograph plane of the region of the $z$-plane shown in Figure 1. The corresponding $\bar{\omega}$-plane is shown in Figure 2(b). The mapping onto the complex potential $z$-plane is shown in Figure 2(c). The auxiliary $\zeta$-plane used in this paper is shown in Figure 2(d). In the case considered by Giger, $F^{\prime}=F=\infty$ in the $z$-plane. In the hodograph plane, $u\left(F^{\prime}\right)=0$. In the potential plane, $\Phi_{e}=\infty$. In the $\zeta$-plane, $s=\infty$. (This problem was also solved by Bear and Dagan [2] in the context of groundwater flow.)

The $f$-plane is mapped onto the $\zeta$-plane via the transformation

$$
f=\frac{Q}{2 \pi} \log \zeta
$$

Here, $Q$ is the strength of the sink and, using the conventions of this paper, has the dimensions of a flow rate per unit length divided by a specific gravity, i.e.,

$$
[Q]=[L]^{4}[T][M]^{-1} .
$$

The $\bar{\omega}$-plane is mapped onto the $\zeta$-plane via

$$
\bar{\omega}=\frac{2}{\pi} \operatorname{arcsinh} \zeta^{1 / 2}
$$

Using (10), the expression relating the $z$-plane to the $\zeta$-plane is derived to be

$$
\frac{d z}{d \zeta}=-\frac{Q}{\pi^{2} K}\left(\frac{\operatorname{arcsinh} \zeta^{1 / 2}}{\zeta}\right)
$$

The shape of the interface $\overline{G F}$ is found by integrating (14) for $-\infty \leq \zeta \leq-1$. If we let $\zeta=\sinh ^{2}(t+i \pi / 2), 0 \leq t \leq \infty$, then the parametric equations of the interface will take the form

$$
\begin{aligned}
& \frac{x K}{Q}=\frac{2}{\pi^{2}} \int_{0}^{\tau} d t t \tanh t, \\
& \frac{y K}{Q}=-\frac{1}{\pi} \log \cosh \tau .
\end{aligned}
$$

The critical height $b$ from the top of the cusp to the sink is obtained by integrating (14) for $-1 \leq \zeta \leq 0$. It is given by

$$
\frac{b K}{Q}=\frac{1}{\pi} \log 2=0.2206 \text {. }
$$


Giger shows that, at large distances from the well, the shape of the interface is given by the equation of a parabola

$$
y^{2}\left(\frac{\log 2}{b}\right)^{2}-x \pi\left(\frac{\log 2}{b}\right)-\frac{\pi^{2}}{12}=0 .
$$

This result is obtained using the approximation of uniform linear flow in the reservoir, the so-called "Dupuit-Forcheimer approximation" [5]. In this approximation, the pressure drop in the flowing oil due to the vertical component of the velocity vector is omitted. It is valid at large distances from the well but breaks down near the well where the flow lines deviate from the horizontal.

To find the height $h$ of the reservoir, the crest is simply truncated at the outer radius, i.e. $y=h$ at $x=x_{e}$. Having determined a height for the reservoir, we can non-dimensionalise the variables of the problem, setting $z^{*}=z / h$, $x_{e}^{*}=x_{e} / h, b^{*}=b / h$, and $Q^{*}=Q / K h$. Note that the parameter $h$ used here is a specified cutoff and does not correspond to $H$ in Figure 1. Making these changes of variables in (18) and solving the quadratic equation, we obtain

$$
b^{*}=\frac{2 \log 2}{\pi}\left[x_{e}^{*}+\sqrt{\left(x_{e}^{*}\right)^{2}+\frac{1}{3}}\right]^{-1} .
$$

In addition, from (17), we have

$$
b^{*}=Q^{*} \frac{1}{\pi} \log 2 .
$$

Hence, we derive the following relation for the critical rate

$$
Q^{*}=2\left[x_{e}^{*}+\sqrt{\left(x_{e}^{*}\right)^{2}+\frac{1}{3}}\right]^{-1} .
$$

Empirically, for large $x_{e}^{*}$, the values calculated using this relation can be very closely fitted to a curve of the form

$$
Q^{*}=c_{1} x_{e}^{* c_{2}}
$$

where (using linear regression for values calculated with $10<x_{e}^{*}<1000$ ),

$$
c_{1}=0.9997 \text { and } c_{2}=-1.000 \text {. }
$$

Hence, Giger's approach predicts an approximately inverse relationship between $Q^{*}$ and $x_{e}^{*}$, in close agreement with the results of the Dupuit-Forcheimer approximation, which gives an exact inverse relationship. The result is dominated by the uniform linear flow in the larger part of the reservoir. 
In Chaperon's approach [3], the general form of the flow potential is derived from a solution given by Houpert [7] for the case of an infinite row of vertical wells

$$
\phi\left(x^{*}, y^{*}\right)=Q^{*} \frac{1}{2 \pi} \log \left(\cosh \pi x^{*}-\cos \pi y^{*}\right) .
$$

This form of the potential does not take the presence of the crest into account, but it does combine the prevailing characteristics of radial flow near the well with linear flow far from the well. It is used together with the stability condition for the crest, which requires that the potential drop due to the viscous flow be equal to the gravity potential difference, and the condition of dynamic equilibrium, which requires that the buoyancy forces be greater than the viscous forces, to derive an implicit equation for the critical height of the crest

$$
\left(1-b^{*}\right)=\frac{1-\cos \pi b^{*}}{\pi \sin \pi b^{*}} \log \left(\frac{1+\cosh \pi x_{e}^{*}}{1-\cos \pi b^{*}}\right)
$$

and the critical rate

$$
Q^{*}=2 \frac{1-\cos \pi b^{*}}{\sin \pi b^{*}} .
$$

If (25) and (26) are solved to give $Q^{*}$ for various values of $x_{e}^{*}$, then a very close fit of the form (22) can be made to the data pairs with

$$
c_{1}=1.443 \quad \text { and } \quad c_{2}=-0.9439 .
$$

Modifications introduced by Dikken [4] include the effects of the pressure gradient corrections caused by the presence of the crest. Then a good fit is obtained with

$$
c_{1}=0.9437 \text { and } c_{2}=-0.9896 .
$$

Again, this implies an approximate inverse relationship between the critical rate and the outer radius.

\section{Improved model}

In this section, the hodograph solution for the lateral edge drive model with a constant potential boundary at a finite outer radius is given. The shape of the water crest and an expression for the critical rate are calculated. The water crest intersects the constant potential boundary with a horizontal asymptote [1], and the reservoir has a well-defined height ( $H$ in Figure 1$)$ which is the distance 
from this asymptote to the impermeable plane. In the conformal mapping of the $f$ - and $\omega$-planes onto the $\zeta$-plane, the points $F$ and $F^{\prime}$ in the $f$-plane are now at a finite potential, $\Phi_{e}$; and the velocity at $F^{\prime}, u\left(F^{\prime}\right)$, is finite in the $\omega$-plane. This introduces a parameter, $s$, into the hodograph solution, as indicated in Figure 2(d).

If $y_{e}^{*}=y_{e} / H$ is the dimensionless height of the crest above the horizontal asymptote, and $b^{*}=b / H$ is the dimensionless height from the top of the cusp to the sink, then clearly

$$
b^{*}+y_{e}^{*}=1 .
$$

The hodograph method can be used to express $b^{*}, y_{e}^{*}$, and $x_{e}^{*}$ in terms of $Q^{*}$ and the parameter $s$ in the $\zeta$-plane (defined below). The relation (29) then enables a relation for $Q^{*}$ to be derived in terms of $x_{e}^{*}$.

Using the Schwarz-Christoffel transformation, the $f$-plane is mapped onto the $\zeta$-plane via the transformation

$$
f(\zeta)=\frac{Q}{2 \pi} \log \frac{2 s-\zeta-2 \sqrt{s(s-\zeta)}}{\zeta}+\Phi_{e}
$$

As in Section 2 , the $\bar{\omega}$-plane is mapped onto the $\zeta$-plane via

$$
\bar{\omega}=(2 / \pi) \operatorname{arcsinh} \zeta^{1 / 2} .
$$

From this transformation, the value of the parameter $s$ can be derived to be

$$
s=\sinh ^{2} \frac{\pi}{2} \frac{K}{u\left(F^{\prime}\right)} .
$$

Thus, $s$ is determined by the velocity at the point $F^{\prime}, u\left(F^{\prime}\right)$, which is in turn determined by the outer radius and the height of the reservoir.

Using (10), the expression relating the $z$-plane to the $\zeta$-plane is derived to be

$$
\frac{d z^{*}}{d \zeta}=\frac{Q^{*}}{\pi^{2}} \frac{\sqrt{s} \operatorname{arcsinh} \sqrt{\zeta}}{\zeta \sqrt{s-\zeta}} .
$$

Notice that as $s \rightarrow \infty$ this reduces to (14), derived in Section 2.

The critical height from the top of the cusp to the sink is obtained by integrating (33) for $-1 \leq \zeta \leq 0$. It is given by

$$
b^{*}=Q^{*} \frac{2}{\pi^{2}} \int_{0}^{1} d t \frac{t \cot t}{\sqrt{1+\sin ^{2}(t / s)}}
$$


As $s$ becomes large, this quickly converges to the value obtained in Section 2 (c.f. (17) and (20)). This behaviour is illustrated in Table 1, in which values of the integral (34) are given as a function of $s$.

The shape of the interface $\overline{G F}$ is found by integrating (33) for $-\infty \leq \zeta \leq-1$. If we let $\zeta=\sinh ^{2}\left(t+i \frac{\pi}{2}\right), 0 \leq t \leq \infty$, then the parametric equations of the interface will take the form

$$
\begin{gathered}
x^{*}=Q^{*} \frac{2}{\pi^{2}} \int_{0}^{\tau} d t \frac{t \tanh t}{\sqrt{1+\cosh ^{2}(t / s)}}, \\
y^{*}=-Q^{*} \frac{2}{\pi^{2}} \int_{0}^{\tau} d t \frac{\tanh t}{\sqrt{1+\cosh ^{2}(t / s)}} .
\end{gathered}
$$

The $y$-integral (36) can be worked out analytically and is given by

$$
\frac{y^{*}}{Q^{*}}=-\frac{1}{2 \pi} \log \left(\frac{\sqrt{1+\cosh ^{2}(\tau / s)}-1}{\sqrt{1+\cosh ^{2}(\tau / s)}+1}\right) .
$$

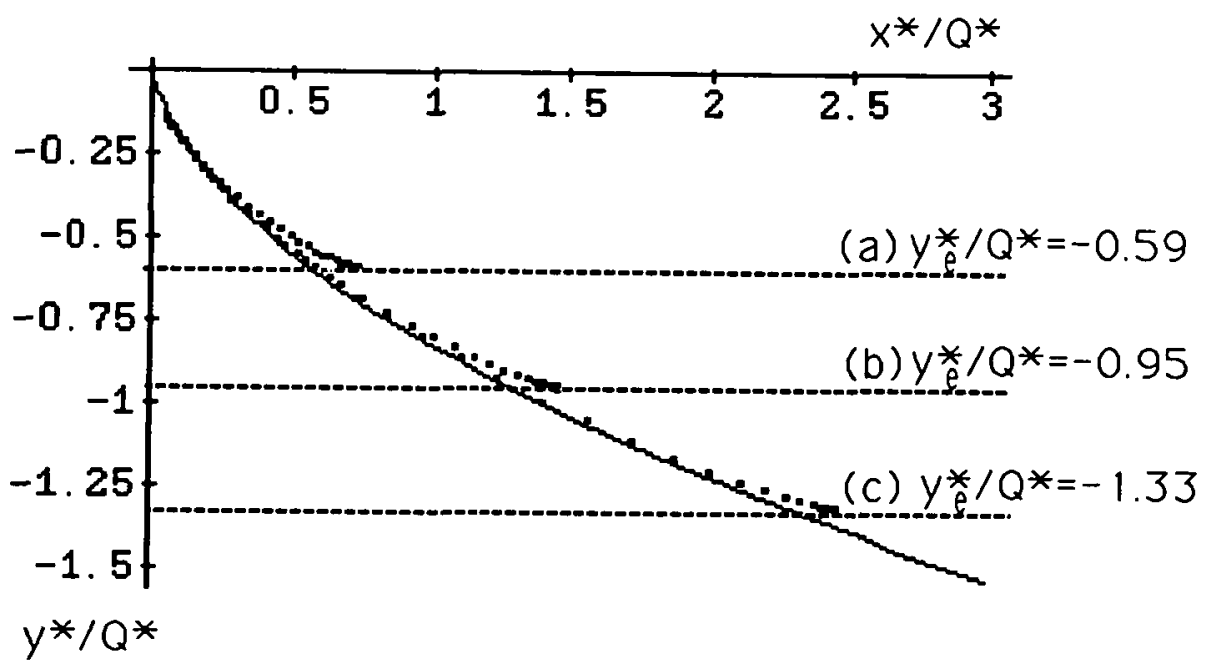

FIGURE 3. The free interfaces calculated using the hodograph method with (a) $s=10$, (b) $s=100$ and (c) $s=1000$. Also shown is the limiting free interface obtained as $s \rightarrow \infty$. 


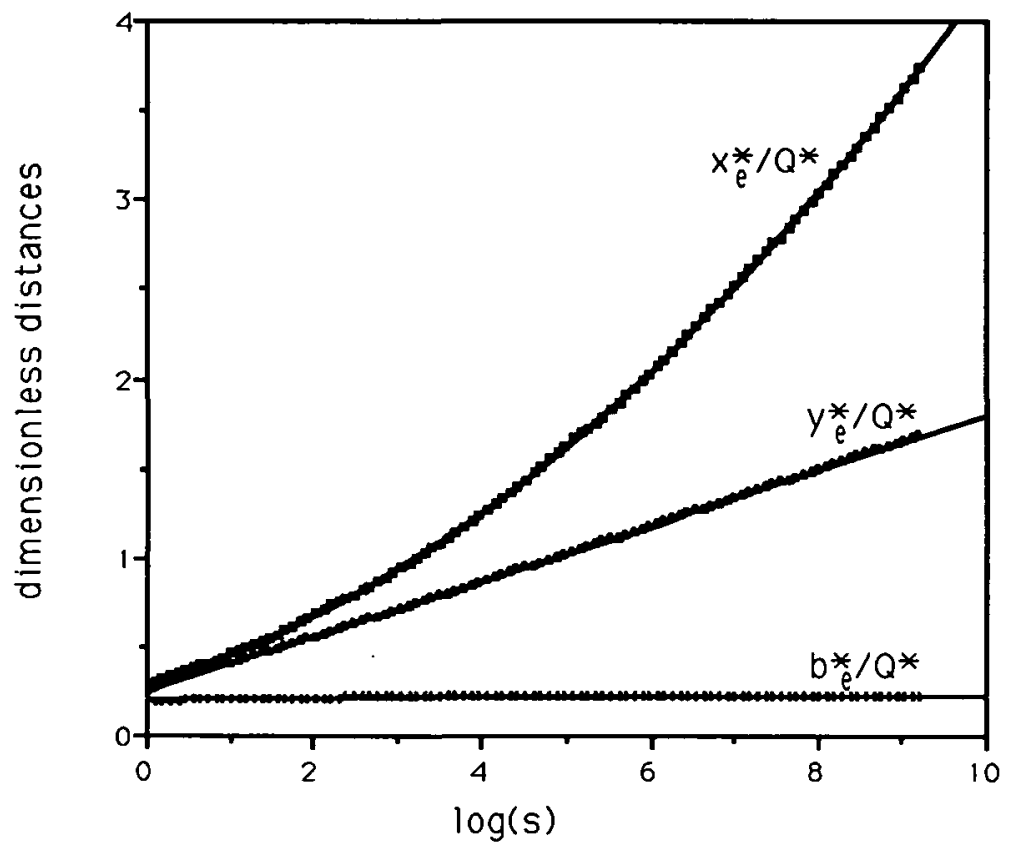

FIGURE 4. Calculated values of the dimensionless distances $x_{e}^{*} / Q^{*}, y_{e}^{*} / Q^{*}$ and $b_{e}^{*} / Q^{*}$ as a function of $\log s$, compared with the fitted curves.

In particular, note that the value of the integral is finite as $\tau \rightarrow \infty$ and is given by

$$
\frac{y_{e}^{*}}{Q^{*}}=-\frac{1}{2 \pi} \log \left(\frac{\sqrt{1+1 / s}-1}{\sqrt{1+1 / s}+1}\right) .
$$

Figure 3 shows the free interface calculated using various values of the parameter $s$, compared with the limiting free interface obtained when $s \rightarrow \infty$, which forms an envelope for the solutions. The values of $y_{e}^{*}$ are written on the figure.

A good approximation to $y_{e}^{*}$ for large $s$ is given by

$$
\frac{y_{e}^{*}}{Q^{*}} \approx \frac{1}{2 \pi} \log (4 s)=\frac{1}{\pi}\left(\log 2+\frac{1}{2} \log s\right) .
$$

This log-linear fit is compared to the exact curve in Figure 4. Also, Table 1 demonstrates how the fit improves for increasing $s$. 


\begin{tabular}{|l|l|l|l|l|l|}
\hline$s$ & $\begin{array}{l}b^{*} / Q^{*} \\
\text { (comp) }\end{array}$ & $\begin{array}{l}x_{e}^{*} / Q^{*} \\
\text { (comp) }\end{array}$ & $\begin{array}{l}x_{e}^{*} / Q^{*} \\
\text { (fit) }\end{array}$ & $\begin{array}{l}y_{e}^{*} / Q^{*} \\
\text { (exact) }\end{array}$ & $\begin{array}{l}y_{e}^{*} / Q^{*} \\
\text { (fit) }\end{array}$ \\
\hline 1.096 & 0.1945 & 0.3102 & 0.3232 & 0.2910 & 0.2353 \\
2.290 & 0.2061 & 0.4252 & 0.4438 & 0.3827 & 0.3526 \\
4.786 & 0.2131 & 0.5689 & 0.5920 & 0.4853 & 0.4698 \\
10.00 & 0.2168 & 0.7591 & 0.7677 & 0.5948 & 0.5871 \\
20.89 & 0.2188 & 0.9428 & 0.9709 & 0.7081 & 0.7044 \\
43.65 & 0.2197 & 1.171 & 1.202 & 0.8235 & 0.8216 \\
100.0 & 0.2202 & 1.482 & 1.494 & 0.9544 & 0.9536 \\
208.9 & 0.2205 & 1.753 & 1.783 & 1.071 & 1.071 \\
436.9 & 0.2205 & 2.070 & 2.100 & 1.188 & 1.188 \\
1000 & 0.2206 & 2.477 & 2.489 & 1.320 & 1.320 \\
10000 & 0.2206 & 3.742 & 3.752 & 1.687 & 1.687 \\
\hline
\end{tabular}

TABLE 1. Comparison between the calculated and fitted values of the dimensionless distances $b^{*} / Q^{*}, x_{e}^{*} / Q^{*}$ and $y_{e}^{*} / Q^{*}$ for a range of values of the parameter $s$.

The $x$-integral (35) cannot be worked out analytically. However, an approximation can be made for the definite integral at $\tau=\infty$, denoted by $I$, in the manner shown below.

$$
\begin{aligned}
I & =\frac{\pi^{2}}{2} \frac{x_{e}^{*}}{Q^{*}}=\int_{0}^{\infty} d t \frac{t \tanh t}{\sqrt{1+\cosh ^{2}(t / s)}} \\
& =\int_{s^{-1 / 2}}^{\infty} d x \frac{\log \left(\sqrt{s} x+\sqrt{s x^{2}-1}\right)}{x \sqrt{1+x^{2}}} \\
& =\frac{1}{2} \log s \int_{s^{-1 / 2}}^{\infty} d x \frac{1}{x \sqrt{1+x^{2}}}+\int_{s^{-1 / 2}}^{\infty} d x \frac{\log x}{x \sqrt{1+x^{2}}} \\
& \quad+\int_{s^{-1 / 2}}^{\infty} d x \frac{\log \left(1+\sqrt{1-\left(s x^{2}\right)^{-1}}\right)}{x \sqrt{1+x^{2}}} \\
& =I_{1}+I_{2}+I_{3} .
\end{aligned}
$$

Analysis of these integrals, by the means of asymptotic expansions, leads to the 
following results:

$$
\begin{gathered}
I_{1}=\frac{1}{4}(\log s)^{2}+\frac{1}{2} \log 2 \log s+\mathrm{O}(1), \\
I_{2}=-\frac{1}{8}(\log s)^{2}+\mathrm{O}(1), \\
I_{3}=\frac{1}{2} \log 2 \log s+\mathrm{O}(1)
\end{gathered}
$$

where the $O(1)$ terms, i.e. definite integrals which are $O(1)$ as $s \rightarrow \infty$, have been omitted for clarity. Combining these results, we find that $I$ is given by

$$
I=(\log s)^{2} / 8+\log 2 \log s+C
$$

where $C$ is an $O(1)$ term which can be shown to be

$$
C=\int_{0}^{1} d x \frac{\log 2 x}{x}\left(\frac{1-\sqrt{1+x^{2}}}{\sqrt{1+x^{2}}}\right)+\int_{1}^{\infty} d x \frac{\log 2 x}{x \sqrt{1+x^{2}}} \approx 1.48
$$

Hence, we derive the approximate expression

$$
\frac{x_{e}^{*}}{Q^{*}} \approx \frac{2}{\pi^{2}}\left(\frac{1}{8}(\log s)^{2}+\log 2 \log s+1.48\right) .
$$

This approximation is very good for $s>1$, as is demonstrated by comparison with the computed values of (35), given in Table 1 . The fit improves as $s$ increases (see also Figure 4).

Using (29), together with (20) and (39), we derive an implicit equation for $Q^{*}$ :

$$
Q^{*} \approx \pi\left[2 \log 2+\frac{1}{2} \log s\left(Q^{*}\right)\right]^{-1}
$$

Also, (46) is a quadratic which can be solved to give $\log s$ as a function of $x_{e}^{*} / Q^{*}$. Then, substituting in (47) and performing the algebra we get

$$
\begin{aligned}
Q^{*} & =2\left[x_{e}^{*}+\sqrt{\left(x_{e}^{*}\right)^{2}+\frac{8}{\pi^{2}}\left(2(\log 2)^{2}-C\right)}\right] \\
& \approx 2\left[x_{e}^{*}+\sqrt{\left(x_{e}^{*}\right)^{2}-0.42}\right] .
\end{aligned}
$$

This should be compared with (21) in Section 2. 
The fit given by (48) will be very accurate for large $x_{e}^{*}$. From Table 1 , it is clear that the various approximations made to the integrals for $b^{*}, y_{e}^{*}$, and $x_{e}^{*}$, derived using the hodograph method, are already very good when $s=200$, which corresponds to $x_{e}^{*} \approx 1.4$. Therefore, $(48)$ can be applied for all practical purposes in oil reservoirs (in which, typically, $x_{e}^{*}>5$ ).

The values calculated using (48) can be fitted to a curve of the form (22) where (using linear regression for values calculated with $10<x_{e}^{*}<1000$ ),

$$
c_{1}=1.000 \text { and } \quad c_{2}=-1.000
$$

Hence, to four-figure accuracy, an inverse relationship with $x_{e}^{*}$ is obtained.

A comparison of the critical rates calculated using Giger (21), Dikken (28), and the relation derived in this paper (48) are given in Table 2. The three approaches give values which are quantitatively very close and which tend to an inverse relationship with $x_{e}^{*}$ as $x_{e}^{*} \rightarrow \infty$, as predicted using the DupuitForcheimer approximation. The main advantage of the approach taken in this paper, therefore, is not a correction to the quantitative predictions of the critical rate, but the removal of physical inconsistencies in the underlying theory which have led to its rejection in the past.

\begin{tabular}{|l|l|l|l|}
\hline$x_{e}^{*}$ & $Q^{*}$ & $Q^{*}[6]$ & $Q^{*}[4]$ \\
\hline 1. & 1.1270 & 0.9282 & 0.9437 \\
2. & 0.5132 & 0.4900 & 0.4753 \\
3. & 0.3371 & 0.3303 & 0.3182 \\
4. & 0.2516 & 0.2487 & 0.2494 \\
5. & 0.2008 & 0.1993 & 0.1919 \\
10. & 0.1001 & 0.1000 & 0.0967 \\
20. & 0.0500 & 0.0500 & 0.0487 \\
40. & 0.0250 & 0.0250 & 0.0245 \\
60. & 0.0167 & 0.0167 & 0.0164 \\
80. & 0.0125 & 0.0125 & 0.0124 \\
100. & 0.0100 & 0.0100 & 0.0099 \\
\hline
\end{tabular}

TABLE 2. Comparison of critical rates calculated using the fit derived in this paper and those derived by Giger [6] and Dikken [4]. 


\section{Conclusion}

In this paper an improved model for water cresting has been presented. It is based on the standard lateral edge drive model but corrects the physical inconsistencies which have plagued that model due to the incorrect implementation of boundary conditions in previous analytical treatments. Previous treatments, by Giger and Chaperon, led to the unphysical result of a water-oil interface which does not approach a horizontal asymptote at a large distance from the well but, rather, tends to a parabolic curve. This meant that, in order to introduce a height for the reservoir and to calculate critical rates, the interface had to be truncated at the outer radius, intersecting the initial water-oil contact at a non-zero angle. The physical inconsistencies in the theory led to skepticism about the ability of the model to predict useful quantitative results. It has generally been recommended that one avoid the use of the model for any purpose other than a study of the local deformation of the water-oil contact below the horizontal well.

By using the hodograph method with a constant potential boundary at the outer radius we have ensured that the interface tends to a horizontal asymptote. The hodograph solution yields the shape of the free interface. It also yields integral representations for the lengths of boundary segments, and these have enabled us to derive an expression for the critical rate. The critical rates calculated using the method of this paper do not differ significantly from those calculated using previous approaches. The main advantage of the improved model, therefore, is not a correction to the quantitative predictions of the critical rate, but the removal of physical inconsistencies in the underlying theory.

\section{Acknowledgements}

I would like to thank Dr M.J. Landman for his help with the asymptotic expansions in Section 3. I also thank The Broken Hill Proprietary Co. Ltd. for permission to publish this paper.

\section{References}

[1] J. Bear, Dynamics of fluids in porous media (American Elsevier, New York, 1972).

[2] J. Bear and G. Dagan, "Some exact solutions of interface problems by means of the hodograph method”, J. Geophys. Res. 69 (1964) 1563-1572. 
[3] I. Chaperon, "Theoretical study of coning towards horizontal and vertical wells in anisotropic formations: subcritical and critical rates", SPE (Society of Petroleum Engineers), Paper No. 15377 (October 5 1986) .

[4] B. J. Dikken, "Pressure drop in horizontal wells and its effect on their production performance", SPE (Society of Petroleum Engineers), Paper No. 19824 (October 8 1989).

[5] J. Dupuit, Etudes théoriques et pratiques sur le mouvement des eaux, 2nd ed. (Dumont, Paris, 1863).

[6] F. M. Giger, "Analytic 2-D models of water cresting before breakthrough for horizontal wells”, SPE Res. Eng. (November 1989) 409-416.

[7] A. Houpeurt, Eléments de mécanique des fluides dans les milieux poreux (Editions Technip, Paris, 1975).

[8] J. McCarthy, "Gas and water cresting towards horizontal wells", J. Aust. Math. Soc. Ser. B 35 (1993) 174-198.

[9] M. Muskat and R. B. Wyckoff, "An approximate theory of water coning in oil production", Trans. AIME 114 (1935) 144-163. 